
\title{
CIÊNCIA'NATURA
}

\section{Avaliação de modelos de footprint para análise de fluxos obtidos por eddy-covariance em pequenas-áreas.}

Evaluation of footprint models for fluxes analysis using the eddy-covariance technique over small areas.

Ivan Mauricio Cely Toro, Ricardo Acosta Gotuzzo, Débora Regina Roberti e Jackson Ernani Fiorin

Universidade Federal de Santa Maria, Santa Maria, RS, Brasil

\section{Resumo}

São comparados dois modelos de cálculo do footprint de medidas de fluxo superficiais. Os cálculos estão baseados nos modelos analíticos de footprint de Kormann e Meixner (2001) [An analytical footprint model for non-neutral stratification. Boundary-Layer Meteorology 99, 207-224] e de Schuepp et al. (1990) [Footprint prediction of scalar fluxes from analytical solutions of the difussion equation. Boundary-Layer Meteorology 50, 355-373]. As funções de densidade do footprint de um sensor de fluxo são determinadas utilizando os dados obtidos pelo método de eddy-covariance. Estas funções são integradas acima de áreas dadas como polígonos quadrangulares, como o caso campos de agricultura. Neste trabalho observam-se as características de cada modelo de footprint fazendo uso das medidas de fluxo com um sistema de eddy-covariance da rede SULFLUX, instalado em uma área de cultura agrícola. Finalmente se discute qual modelo descreve de maneira mais precisa as medições de fluxo em pequenas áreas.

Palavras-chave: Footprint, eddy-covariance, medidas de fluxo.

\section{Abstract}

Two models for footprint calculations are compared employing flux measurements in the planetary boundary layer. The calculations are based on the analytical models by Kormann e Meixner (2001) [An analytical footprint model for non-neutral stratification. Boundary-Layer Meteorology 99, 207-224] and by Schuepp et al. (1990) [Footprint prediction of scalar fluxes from analytical solutions of the difussion equation. Boundary-Layer Meteorology 50, 355-373]. The footprint density functions of a flux sensor are determined using eddy-covariance data. Those functions are integrated over surfaces given by quadrangular rectangles, in this case an agricultural field. This work ilustrates the features of each footprint model employing flux measurements with an eddy-covariance system of the SULFLUX network, installed on a agricultural field. Finally, it is presented the model that describes in a better way the flux measurements in small fields.

Keywords: Footprint, eddy-covariance, flux measurements. 


\section{Introdução}

O estudo dos fluxos superficiais de energia, água e carbono tem sido cada vez mais utilizados para entender as interações biosfera-atmosfera em uma situação evidente de mudanças climáticas. A técnica de eddy-covariance (EC) tem sido amplamente empregada para obter medidas de fluxos superficiais, que pode ser aplicada ao monitoramento das emissões de gases do efeito estufa $(\mathrm{GHG})$ tais como $\mathrm{CO}_{2}$ e $\mathrm{CH}_{4}$, diretamente relacionados com o aquecimento global. A técnica EC necessita de medição de alta frequência, usualmente obtidas em torres micrometeorológicas.

A técnica de EC permite estimar fluxos sobre grandes áreas, diferentemente das câmaras de medição de fluxo, pois nestas, as informações obtidas estão confinadas em uma área fixa delimitada pelas paredes da mesma. Por outro lado, na técnica de EC a área que contribui significativamente nas medidas do fluxos superficiais é uma função complexa que precisa ser avaliada em relação a: (i) altura em que são realizadas as medidas; (ii) rugosidade da superfície; (iii) altura e estrutura da cobertura; (iv) junto com condições micrometeorológicas (velocidade e direção do vento, intensidade da turbulência e estabilidade atmosférica entre outras).

O termo footprint define o "campo de visão"do sensor, ou seja, o contexto espacial de uma medida de fluxo superficial. Segundo Vesala et al. (2008), o footprint é o cálculo (nem sempre simples) necessário para conhecer a contribuição relativa de cada elemento de área na medição de fluxos verticais ou concentrações. É possível abordar este problema numericamente, com duas aproximações diferentes: (1) Métodos convencionais deterministas ou modelos analíticos, baseados na equação semi-empírica de difusão turbulenta, suposições de fechamento ou outros modelos de filtragem como grandes simulações, e (2) modelos estocásticos, baseados tanto em simulações do campo Euleriano de velocidades aleatórias ou modelos lagrangianos baseados em simulações de trajetórias lagrangianas.

Neste trabalho, comparam-se dois modelos analíticos de predição do footprint sobre uma cultura de aveia (Avena Sativa) no sul do Brasil, na região de Cruz Alta - RS. Por se tratar de uma área experimental de comparação de diferentes manejos do solo, a cultura avaliada foi plantada em uma parcela experimental de $40 \mathrm{~m} x 60 \mathrm{~m}$. No entanto, os fluxos superficiais podem ser afetados por uma região maior do que a área do plantio, interferindo na real representação da cultura a ser estudada. A importância em se fazer um estudo sobre a comparação de modelos para calcular o fooprint Foken e Leclerc (2004), reside na tentativa de explicar qual dos modelos descreve de maneira melhor adequada a contribuição do sinal oriunda da vegetação de interesse, para o posterior tratamento e análises dos fluxos medidos em pequenas áreas de cultura. Waldo et al. (2016).

\section{Metodologia}

\subsection{Cálculo do footprint}

A definição fundamental da função de footprint $\phi$ está dada pela integral da equação de difusão Pasquill (1972). Onde o fluxo turbulento vertical $F\left(0,0, z_{m}\right)$, medido na origem de um sistema horizontal de coordenadas $(x=0, y=0)$, na altura $z_{m}$ está relacionado com o correspondente fluxo superficial $F\left(x, y, z_{m}\right)$ (razão de emissão fonte ou sumidouro) contra a direção do vento da medida pontual por:

$$
F\left(0,0, z_{m}\right)=\int_{-\infty}^{\infty} \int_{0}^{\infty} F(x, y, 0) \phi\left(x, y, z_{m}\right) d x d y
$$

Onde o eixo $x$ aponta na direção contrária à velocidade média horizontal do vento. A já nomeada função footprint $\phi(x, y, z)$ descreve a influência de uma unidade pontual de fonte em qualquer localização da superfície $(x, y)$ do fluxo medido em $(0,0, z)$. A tabela 1 resume os termos empregados neste trabalho.

\subsubsection{Modelo de Kormann e Meixner (2001)}

De acordo com Neftel et al. (2008), o marco de referência matemático deste modelo é baseado na formulação de um gradiente de difusão estacionário (2),

$$
f(x, z)=-\frac{K}{\bar{u}} \frac{\partial D_{z}}{\partial z}=-K \frac{\partial c(x, z)}{\partial z}
$$

assumindo a não dependência da altura com a dispersão do vento lateral (3)

$$
u \frac{\partial c}{\partial x}=-\frac{\partial f}{\partial z}
$$

A chave para obter a solução analítica da função footprint é o uso da lei de potências para a distribuição vertical dos perfis de velocidade do vento (4a) e de difusividade turbulenta(4b)

$$
u(z)=U z^{m}
$$


Tabela 1: Notação.

\begin{tabular}{|c|l|}
\hline Simbolo & Quantidade \\
\hline$c(x, z)$ & Concentração integrada do vento lateral \\
$d$ & Plano de deslocamento zero \\
$D_{y}$ & Função de distribuição do vento lateral \\
$f(x, z)$ & Fluxo de footprint do vento lateral \\
$F(x, y, z)$ & Fluxo \\
$k$ & Constante de von kárman $(k=0.4)$ \\
$K(z)$ & Perfil vertical de difusividade turbulenta \\
$L$ & Comprimento de Obukhov \\
$m$ & Expoente da lei de potências da velocidade do vento \\
$n$ & Expoente da lei de potências da difusividade turbulenta \\
$r+m-n$ & Fator de forma \\
$U$ & Constante na lei de potências do perfil de difusividade turbulenta \\
$u_{\star}$ & Velocidade de atrito \\
$\bar{u}(x)$ & Velocidade efetiva da pluma \\
$z_{m}$ & Altura da medida \\
$z(x)$ & Altura média da pluma \\
$\kappa$ & Constante na lei de potências da velocidade do vento \\
$\mu=(1+m) / r$ & Constante \\
$\xi(z)$ & Comprimento de escala de fluxo \\
$\sigma$ & Dispersão do vento lateral \\
$\phi(x, y, z)$ & Fluxo de footprint \\
$\Gamma(x)$ & Função Gamma \\
\hline
\end{tabular}

$$
K(z)=\kappa z^{n}
$$

a equação da concentração do vento lateral integrado (por uma fonte deunidade pontual) (5)

$$
c(x, z)=\frac{A}{\overline{z u}} \exp \left[-\left(\frac{B z}{\bar{z}}\right)^{r}\right]
$$

definindo o comprimento de escala do fluxo e $\xi(u)=\frac{U z^{r}}{r^{2} k}$ e a constante $u=(1+m) / r$, então a equação (5) pode ser reescrita como

$$
c=\frac{1}{\Gamma(\mu)} \frac{r}{U z^{(1+m)}} \frac{\xi^{\mu}}{x^{\mu}} e^{\frac{-\xi}{x}}
$$

Finalmente, juntando a equação (2), com as equações (4a), (4b) e (6), se obtêm

$$
f=\frac{1}{\Gamma(\mu)} \frac{\xi^{\mu}}{x^{1+\mu}} e^{\frac{-\xi}{x}}
$$

que descreve o fluxo do vento lateral integrado na distância a favor do vento $x>0$ e na altura $z$ (contida em $\xi$ ). Tomando a distribuição Gaussiana do vento lateral (8) de Pasquill (1972)

$$
D_{y}(x, y)=\frac{1}{\sqrt{2 \pi} \sigma} e^{\frac{-y^{2}}{2 \sigma^{2}}}
$$

assim se deduze o fluxo de footprint, $\phi(x, y, z)=D_{y} f$ necessários para resolver a equação (1) juntos com as equações (7) e (8).

\subsubsection{Modelo de Schuepp et al. (1990)}

O termo fluxo de footprint foi empregado pela primeira vez por Schuepp et al. (1990). Este estudo foi o primeiro a apresentar um modelo de footprint diferencial definido por (1), para fluxos escalares sob condições de estabilidade neutras.

Como descrito no seu trabalho Kormann e Meixner (2001), a equação (7) inclui a solução particular dada por Schuepp et al. (1990), com o expoente da lei de potências $n=1$ da difusividade turbulenta (4a) para condições de estabilidade neutras e o expoente da lei de potências $m=0$ para a velocidade do vento conjugada. Deste modo, aplicando estas condições dos expoentes nas equações (2), (4a), (4b) e (6) se obtêm: 


$$
f=-\frac{U(z-d)}{u_{\star} k x^{2}} e^{-\frac{U(z-d)}{u_{\star} k x}}
$$

a equação (9) define o footprint unidimensional proposto em seu trabalho por Schuepp et al. (1990). Esta relação explica a contribuição relativa das fontes na direção contrária ao vento a distâncias $x$ das medidas de fluxo no ponto $(x, y)$.

A vantagem desta proposta para predizer o footprint reside na sua simplicidade mecânica e analítica, resultado das suposições acerca da velocidade do vento, por tanto se pode afirmar que serve como uma primeira estimativa para tal predição.

\subsection{Sitio experimental}

O sítio experimental deste estudo está localizado mesorregião do Noroeste Rio-Grandense, na Fundação Centro de Experimentação e Pesquisa (FUNDACEP), Cruz Alta, RS, nas coordenadas: $\left(28^{\circ} 36^{\prime} \mathrm{S}, 53^{\circ} 40^{\prime} \mathrm{W}, 409 \mathrm{~m}\right.$ a.s.1.). O sítio foi estabelecido em 1985, como um centro de pesquisa agrícola cujo objetivo é comparar sistemas de plantio direto (PC) e convencional (PC). A montagem experimental consiste de uma matriz de dez campos ou parcelas, numeradas de 1 até 5 e distribuídas em duas colunas como mostrado Figura 1, as dimensões aproximadas de cada parcela são $40 \mathrm{~m} \times 60 \mathrm{~m}$. Duas torres de fluxo de eddy covariance foram instaladas no centro dos campos número 4, para realizar o monitoramento das trocas de massa e energia entre sistemas de diferentes culturas e a atmosfera.
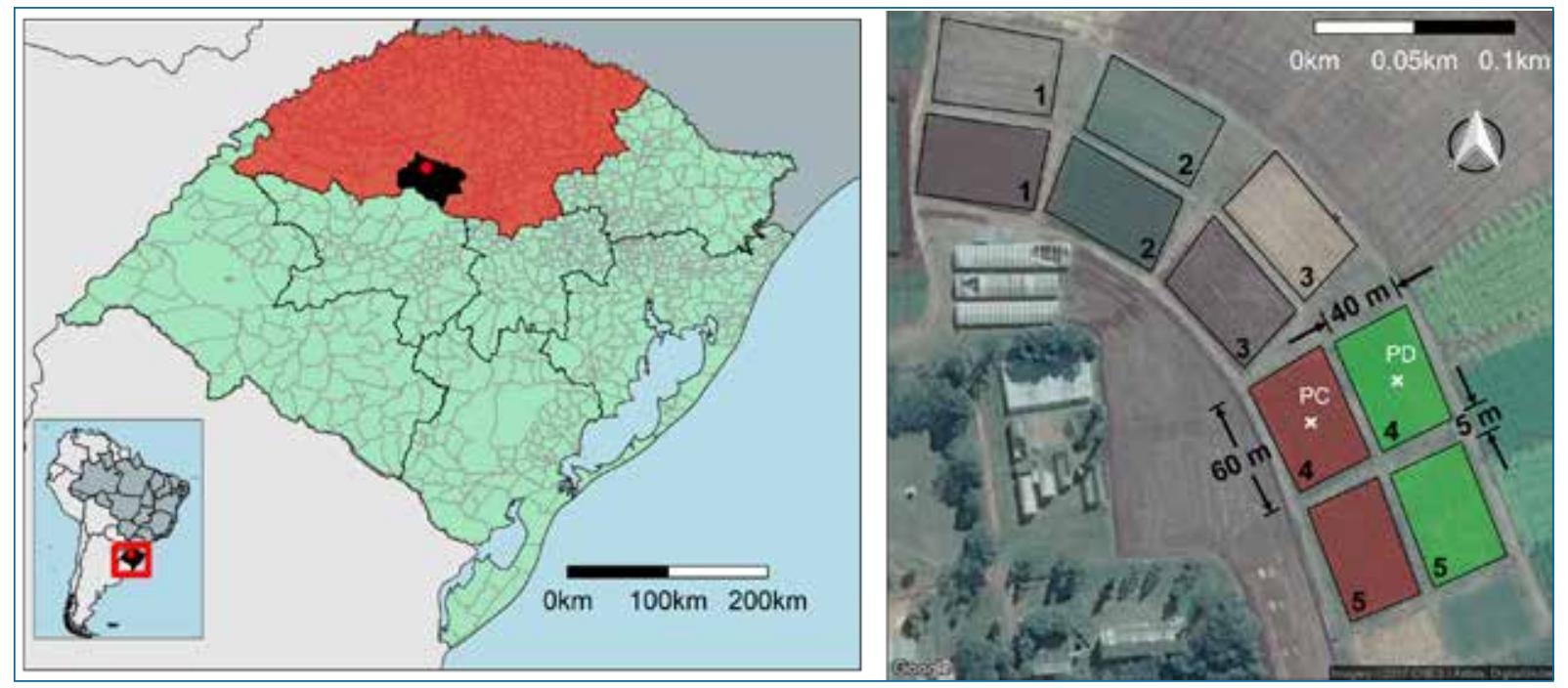

Figura 1: Localização do sítio experimental de Cruz Alta - RS. Os retângulos em verde representam as parcelas com o sistema de plantio direto (PD) e os retângulos em vermelho as áreas com o plantio convencional (PC). As cruzes em branco representam as torres de medição de fluxo.

Para este estudo foram empregados os dados coletados na torre sobre o sistema de plantio direto (PD). O período escolhido para fazer a análise dos modelos foi 15 de Setembro de 2012. As variáveis micrometereológicas foram registradas numa frequência de $10 \mathrm{~Hz}$ e processadas para gerar series de dados cada 30 minutos com o software LI-COR EddyPro ${ }^{\circledR}$, os sensores estavam instalados a $2.5 m$ de altura.

\section{Resultados}

Foram calculadas as médias diária das seguintes variáveis micrometeorológicas obtendo $u_{\star}\left(\mathrm{ms}^{-1}\right)=0.30, z_{m} / L=0$ e $U\left(m s^{-1}=3.05\right)$ para aplicar em cada modelo de footprint. Foi escolhido este dia visto que as condições de estabilidade atmosféricas eram quase neutras, requerimento indispensável para a avaliação do modelo Schuepp et al. (1990). Neste período a altura da cultura era de $62 \mathrm{~cm}$, por tal motivo o plano de deslocamento zero $d$ foi estimado como $2 / 3$ da altura da cultura Garratt (1992). O processamento e análises de dados e estatístico foi feito com a linguagem e ambiente de desenvolvimento integrado $\boldsymbol{R}$ R Core Team (2017). Os dois modelos de footprint foram determinados com o pacote FREddyPro Xenakis (2016), desenhado para o modelagem de footprint após o processamento geral de dados obtidos com sistema EC pelo software $L I$-COR EddyPro ${ }^{\circledR}$

A figura 2, apresenta a pluma do footprint sobre as parcelas dos dois modelos analíticos estudados para condições de estabilidade neutras. É necessário ressaltar a diferença de cada pluma. Na figura 2(a) se observa que é mais "fechada", ou seja grande parte de sua superfície, está delimitada dentro das áreas de cada cultura, por outro lado o inverso ocorre com o modelo 


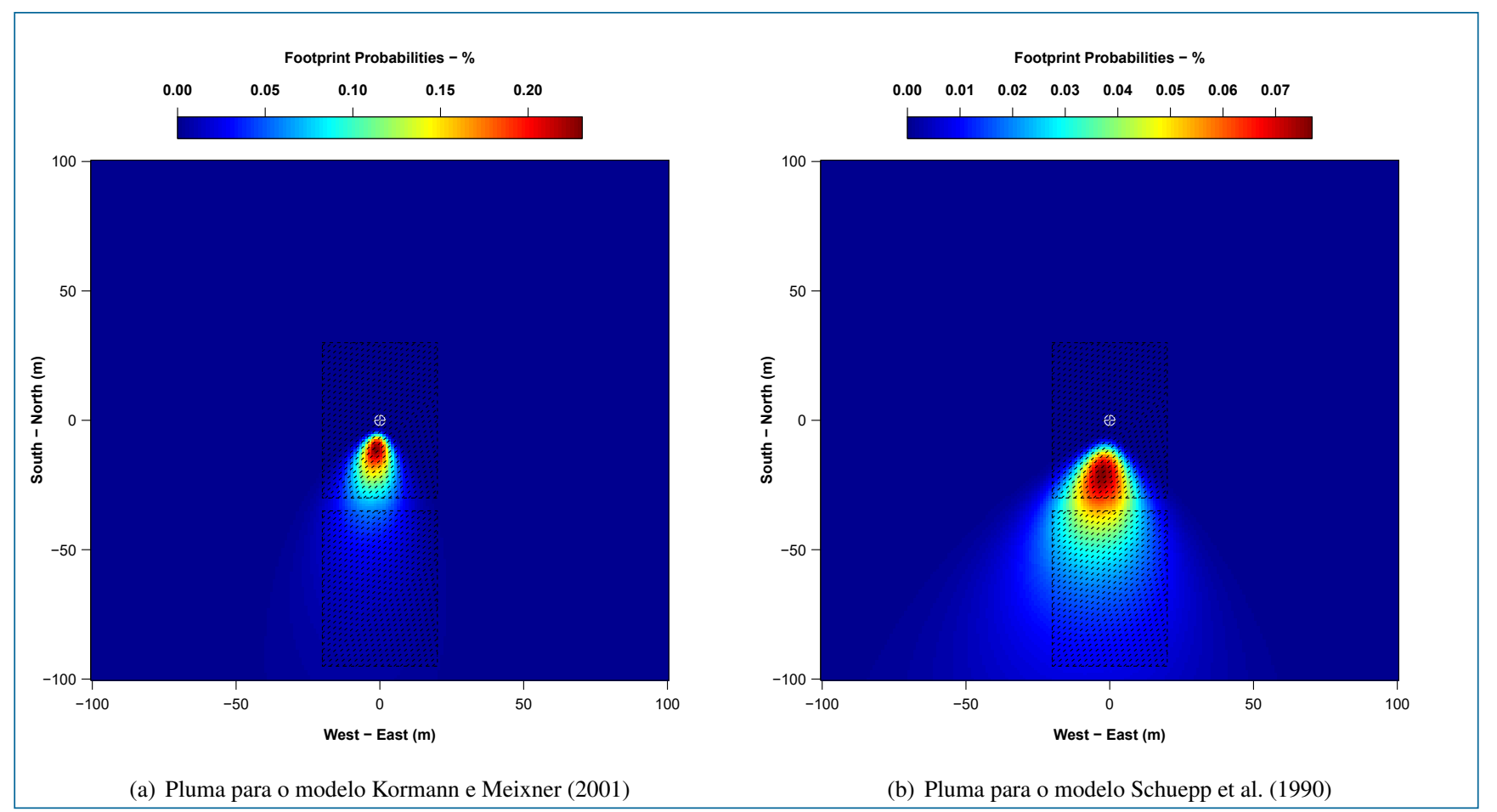

Figura 2: Ilustração da pluma do footprint para cada modelo sobre os dois plantios. As áreas mais vermelhas indicam as distâncias ao sensor onde a contribuição do footprint é mais sensível. As áreas pretas representam as parcelas.

representado na figura 2(b), em que a pluma está mais dispersa. Calculando a contribuição ("área de cada pluma"), contida nos dois retângulos que representam o sinal oriundo das culturas, se obtêm que para o modelo de Kormann e Meixner (2001) a quantidade da pluma nas culturas é de aproximadamente $82 \%$ enquanto para o modelo de Schuepp et al. (1990) é de aproximadamente $69 \%$.

$\mathrm{Na}$ figura 3(a) observa-se a contribuição relativa da superfície ao fluxo para condições de estabilidade neutras para cada modelo. Para o modelo de Kormann e Meixner (2001) se pode ver que o pico do footprint (linha vermelha), que representa a área na qual a contribuição das medições de fluxo é maior, está perto dos $20.35 \mathrm{~m}$, enquanto que, para o modelo de Schuepp et al. (1990) (linha verde) está a $31.15 \mathrm{~m}$ do sensor. Também se analisou que o modelo de Kormann e Meixner (2001) supera em 35\% a contribuição relativa em comparação com o outro modelo, para este caso em particular.

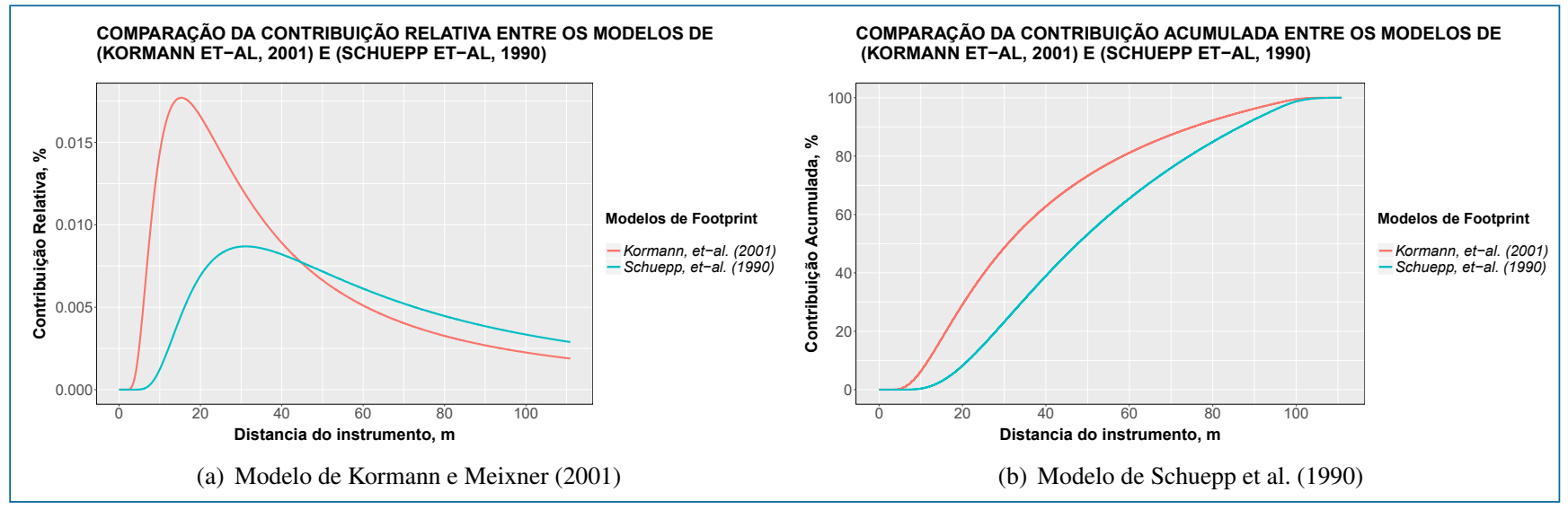

Figura 3: Comparação da contribuição relativa e acumulada entre os modelos de predição do footprint de (Kormann et-al, 2001) e (Schuepp et-al, 1990). Figura 3(a) mostra a contribuição relativa da superfície ao fluxo para condições de estabilidade neutras. Figura 3(b) apresenta contribuição acumulada normalizada como função da distancia em direção oposta ao vento.

Da figura 3(b) se pode inferir a contribuição acumulada para cada método de predição do footprint, tomando $90 \mathrm{~m}$ como a distância do sensor até o extremo inferior do segundo terreno (onde não está a torre), nota-se que para o modelo de Kormann e Meixner (2001) a contribuição acumulada é de 95\% e bem próxima do modelo de Schuepp et al. (1990) com 92\%. Depois calcula-se para o talhão onde está o sensor, (30m desde a torre até o extremo inferior do retângulo) e a contribuição respetiva para 
cada modelo foi $38 \%$ e $23 \%$.

Assim, é possível estabelecer que o modelo de Kormann e Meixner (2001) apresenta certas vantagens sobre o modelo de Schuepp et al. (1990), já que serve como predição do footprint para áreas pequenas, por exemplo áreas de agricultura. Cabe ressaltar também que o modelo de Kormann e Meixner (2001) é aplicável sem importar as condições de estabilidade diferente ao modelo de Schuepp et al. (1990) que unicamente descreve fenômenos em condições de neutralidade como foi abordado neste trabalho. A-pesar da existência de modelos de footprint mais complexos (modelos estocásticos), a aplicação dos modelos aqui analisados é plausível como uma primeira estimativa para áreas com culturas de baixa altura.

\section{Conclusões}

A técnica de eddy-covariance (EC) é de grande importância para obter medidas de fluxos verticais, que pode ser aplicada ao monitoramento das emissões de gases do efeito estufa $(G H G)$. Foram avaliados dois modelos analíticos de footprint com dados obtidos com a técnica de eddy-covariance. Os modelos precisam de parâmetros de entrada que são obtidos pela técnica EC. Se discutem as vantagens sobre os modelos tomando em conta certas variáveis meteorológicas e as propriedades de cada modelo para ser aplicado em áreas pequenas.

Se ressalta a utilidade do modelo de Kormann e Meixner (2001) apesar de considerar certas simplificações próprias dos modelos analíticos, este modelo pode ser usado para estimar o footprint sobre áreas com dosséis de vegetação curtos e além podem ser implementados em procedimentos de controle de qualidade de medições de fluxos obtidos com EC. Em comparação a modelos de footprint mais complexos (modelos estocásticos), os modelos avaliados servem como uma primeira estimativa sobre superfícies especificas. As propriedades de contribuição relativa e acumulada foram discutidas.

\section{Referências}

Foken, T., Leclerc, M. Y. (2004). Methods and limitations in validation of footprint models. Agricultural and Forest Meteorology, $127(3-4), 223-234$.

Garratt, J. R. (1992). The atmospheric boundary layer / J. R. Garratt. Cambridge University Press Cambridge ; New York, URL http://www.loc.gov/catdir/toc/cam031/91034340.html.

Kormann, R., Meixner, F. X. (2001). An analytical footprint model for non-neutral stratification. Boundary-Layer Meteorology, 99(2), 207-224, $0005074 \mathrm{v} 1$.

Neftel, A., Spirig, C., Ammann, C. (2008). Application and test of a simple tool for operational footprint evaluations. Environmental Pollution, 152(3), 644-652.

Pasquill, F. (1972). Some aspects of boundary layer description. Quarterly Journal of the Royal Meteorological Society, 98(417), 469-494.

R Core Team (2017). R: A Language and Environment for Statistical Computing. R Foundation for Statistical Computing, Vienna, Austria, URL https://www.R-project.org/.

Schuepp, P. H., Leclerc, M. Y., MacPherson, J. I., Desjardins, R. L. (1990). Footprint prediction of scalar fluxes from analytical solutions of the diffusion equation. Boundary-Layer Meteorology, 50(1), 355-373, URL https : //doi . org/10.1007/ BF 00120530 .

Vesala, T., Kljun, N., Rannik, Ü., Rinne, J., Sogachev, A., Markkanen, T., Sabelfeld, K., Foken, T., Leclerc, M. Y. (2008). Flux and concentration footprint modelling: State of the art. Environmental Pollution, 152(3), 653-666.

Waldo, S., Chi, J., Pressley, S. N., O’Keeffe, P., Pan, W. L., Brooks, E. S., Huggins, D. R., Stöckle, C. O., Lamb, B. K. (2016). Assessing carbon dynamics at high and low rainfall agricultural sites in the inland Pacific Northwest US using the eddy covariance method. Agricultural and Forest Meteorology, 218-219, 25-36.

Xenakis, G. (2016). FREddyPro: Post-Processing EddyPro Full Output File. URL https: //CRAN.R-project.org/ package=FREddyP ro, $\mathrm{r}$ package version 1.0 . 
Ricardo Acosta Gotuzzo

Universidade Federal de Santa Maria, Santa Maria, RS, Brasil E-mail: ricardo.acosta@gmail.com

Débora Regina Roberti

Universidade Federal de Santa Maria, Santa Maria, RS, Brasil E-mail: debora@ufsm.br

Jackson Ernani Fiorin

Universidade Federal de Santa Maria, Santa Maria, RS, Brasil E-mail: jackson.fiorin@ccgl.com.br 